\title{
$n$-REFLEXIVITY FOR LINEAR SPACES OF OPERATORS
}

\author{
by KUN WOOK CHOI
}

(Received 6 January, 1997)

\begin{abstract}
We discuss the relationship between the $n$-reflexivity of a linear sub-space $\mathcal{S}$ in $\mathcal{B}(\mathcal{H})$, property $\left(\mathbf{A}_{1 / n}\right)$, Class $\mathcal{C}_{0}$ and strictly $n$-separating vectors. We also show that every algebraic operator with property $\left(\mathbf{A}_{2}\right)$ is hyperreflexive.
\end{abstract}

1. Introduction. Let $\mathcal{H}$ be a complex Hilbert space and let $\mathcal{B}(\mathcal{H})$ denote the collection of all bounded linear operators on $\mathcal{H}$. For a linear subspace $\mathcal{S} \subset \mathcal{B}(\mathcal{H})$ the reflexive closure of $\mathcal{S}$ is defined as $\operatorname{Ref} \mathcal{S}=\{T \in \mathcal{B}(\mathcal{H}): T x \in[\mathcal{S} x]$ for all $x \in \mathcal{H}\}$, where $\mathcal{S} x=\{S x: S \in \mathcal{S}\}$ and [.] denotes the closure in the norm topology. A linear subspace $\mathcal{S} \subset \mathcal{B}(\mathcal{H})$ is reflexive if $\mathcal{S}=\operatorname{Ref} \mathcal{S}$. It is easily proved that reflexive subspaces are weakly closed and it is also easy to verify that $\operatorname{Ref} \mathcal{A}=\operatorname{Alglat} \mathcal{A}$ if $\mathcal{A}$ is an algebra containing $I_{\mathcal{H}}$, where lat $\mathcal{A}$ is the lattice of invariant subspace for $\mathcal{A}$, and Alglat $\mathcal{A}=\{T \in \mathcal{B}(\mathcal{H})$ : lat $\mathcal{A} \subset$ lat $T\}$. We recall that a subspace $\mathcal{S}$ of $\mathcal{B}(\mathcal{H})$ is hyperreflexive if there is a $K \geq 1$ such that, for every $T$ in $\mathcal{B}(\mathcal{H})$, $\operatorname{dist}(T, \mathcal{S}) \leq K \sup \{\operatorname{dist}(T x, \mathcal{S} x): x \in \mathcal{H},\|x\| \leq 1\}$. The smallest such $K=K(\mathcal{S})$ is the constant of hyperreflexivity of $\mathcal{S}$. Clearly, hyperreflexivity implies reflexivity, but not vice versa [13]. We say that an operator $T$ is reflexive (hyperreflexive) if the weakly closed algebra $W_{T}$ generated by $I_{\mathcal{H}}$ and $T$ is reflexive(hyperreflexive).

For a vector $x \in \mathcal{H}$ and a linear subspace $\mathcal{S} \subset \mathcal{B}(\mathcal{H})$, we define the evaluation map $E_{x}: \mathcal{S} \rightarrow \mathcal{H}$ by $E_{x}(T)=T x$. A vector $x$ in $\mathcal{H}$ separates $\mathcal{S}$ if $E_{x}$ is injective on $\mathcal{S}$ and a vector $x$ in $\mathcal{H}$ strictly separates $\mathcal{S}$ if $E_{x}$ is bounded below on $\mathcal{S}$. By the open mapping theorem, it is easy to see that $x$ strictly separates $\mathcal{S}$ if and only if $x$ separates $\mathcal{S}$ and $\mathcal{S} x$ is norm closed. We write $\mathcal{S}^{(n)}=\left\{S^{(n)} \in \mathcal{B}\left(\mathcal{H}^{(n)}\right): S \in \mathcal{S}\right\}$ as the $n$-fold ampliation of $\mathcal{S}$, where $S^{(n)}$ is the direct sum of $n$ copies of the operators acting on $\mathcal{H}^{(n)}=\mathcal{H} \oplus \ldots \oplus \mathcal{H} . \mathcal{S}$ is said to be $n$-reflexive $(n$ hyperreflexive) if and only if $\mathcal{S}^{(n)}$ is reflexive(hyperreflexive). $\mathcal{S}$ is $n$-reflexive( $n$-hyperreflexive) implies $S$ is $(n+1)$-reflexive $((n+1)$-hyperreflexive), but the converse does not hold. We say that $\mathcal{S}$ has a strictly $n$-separating vector if $\mathcal{S}^{(n)}$ has a strictly separating vector. It is easy to see that if $\mathcal{S}$ has a strictly $n$-separating vector, then $\mathcal{S}$ has a strictly $(n+1)$-separating vector. For a linear subspace $\mathcal{S} \subset \mathcal{B}(\mathcal{H})$ and a linear subspace $\mathcal{M}$ of $\mathcal{H}$, we define $\pi: \mathcal{S} \mid \mathcal{M}$ by $\pi(S)=S \mid \mathcal{M}$. A linear subspace $\mathcal{M}$ is said to be a strictly separating subspace for $\mathcal{S}$ if there exists $\varepsilon>0$ such that $\|S \mid \mathcal{M}\| \geq \varepsilon\|S\|$, for all $S \in \mathcal{S}$. It is easily seen that $\mathcal{M}$ is a strictly separating subspace for $\mathcal{S}$ if and only if the only member $S \in \mathcal{S}$ satisfying $S(\mathcal{M})=\{0\}$ is $S=0$ and $S \mid \mathcal{M}$ is norm closed.

For vectors $x$ and $y$ in $\mathcal{H}$, we write $x \otimes y$ for the rank one operator defined by $(x \otimes y)(u)=(u, y) x, u \in \mathcal{H}$. Let $\mathcal{S} \subset \mathcal{B}(\mathcal{H})$ be a weak*-closed subspace and $n$ is a positive integer. We say that $\mathcal{S}$ has property $\left(\mathbf{A}_{1 / n}\right)$ if every weak*-continuous functional $\varphi$ on $\mathcal{S}$ can be written as

$$
\varphi=\sum_{i=1}^{n}\left[x_{i} \otimes y_{i}\right] \text { for some } x_{i} \text { and } y_{i} \text { in } \mathcal{H}
$$

Glasgow Math. J. 40 (1998) 385-391. 
Furthermore, $\mathcal{S}$ has property $\left(\mathbf{A}_{1 / n}\right)(r), r \geq 1$ if it has property $\left(\mathbf{A}_{1 / n}\right)$ and if for any $C>r$ the decomposition(1) can be realized with $\sum_{i=1}^{n}\left\|x_{i}\right\|\left\|y_{i}\right\| \leq C\|\varphi\|$. Suppose $m$ and $n$ are cardinal numbers such that $1 \leq m, n \leq \aleph_{0}$. We say that $\mathcal{S} \subset \mathcal{B}(\mathcal{H})$ has property $\left(\mathbf{A}_{m, n}\right)$ provided that for every family $\left\{\varphi_{i j}: 0 \leq i<m, 0 \leq j<n\right\}$ of weak*-continuous functional on $\mathcal{S}$, there exist sequences $\left\{x_{i}: 0 \leq i<m\right\}$ and $\left\{y_{j}: 0 \leq j<n\right\}$ of vectors in $\mathcal{H}$ such that

$$
\varphi_{i j}=\left[x_{i} \otimes y_{j}\right]_{\substack{0 \leq i<m \\ 0 \leq j<m}} .
$$

Furthermore, if $m, n \in \mathbf{N}$ and $r$ is a fixed real number satisfying $r \geq 1$, then $\mathcal{S} \subset \mathcal{B}(\mathcal{H})$ is said to have property $\left(\mathbf{A}_{m, n}\right)(r)$ if for every $s>r$, there exist sequences $\left\{x_{i}\right\}_{0 \leq i<m},\left\{y_{j}\right\}_{0 \leq j<n}$ that satisfy (2) and also satisfy the following conditions:

$$
\left\|x_{i}\right\| \leq\left(s \sum_{0 \leq j<n}\left\|\varphi_{i j}\right\|\right)^{1 / 2}(0 \leq i<m),
$$

and

$$
\left\|y_{j}\right\| \leq\left(s \sum_{0 \leq i<m}\left\|\varphi_{i j}\right\|\right)^{1 / 2}(0 \leq j<n) .
$$

For brevity, we shall denote $\left(\mathbf{A}_{n, n}\right)$ by $\left(\mathbf{A}_{n}\right)$.

In [4], H. Bercovici, C. Foias and C. Pearcy [Proposition 9.16] proved that if a weakly closed subspace $\mathcal{S}$ of $\mathcal{B}(\mathcal{H})$ has property $\left(\mathbf{A}_{1}\right)$, then $\mathcal{S}$ is 3-reflexive. In Section 2 [Theorem 2.3], we prove the following generalization: a weak*-closed subspace $\mathcal{S}$ of $\mathcal{B}(\mathcal{H})$ with property $\left(\mathbf{A}_{1 / n}\right)$ is $(2 n+1)$-reflexive. In Section 3 [Theorem 3.2], we prove that every operator of class $\mathcal{C}_{0}$ is 2-reflexive. It was shown by L. Ding [10, Theorem 2.5] that if $\mathcal{S}$ is a fine dimensional linear subspace of $\mathcal{L}(\mathcal{V})$ and $\mathcal{S}$ has a k-dimensional separating subspace, then $\mathcal{S}$ is $(k+1)$ reflexive. In the last section [Theorem 4.2], we prove that a norm closed linear subspace $\mathcal{S}$ of $\mathcal{B}(\mathcal{H})$ with a strictly $n$-separating vector is $(n+1)$-hyperreflexive. We recover a well-known result as a special case.

2. Property $\left(\mathbf{A}_{1 / n}\right)$. If $\mathcal{S}$ is a WOT-closed subspace of $\mathcal{B}(\mathcal{H})$, then $\mathcal{S}$ is weak ${ }^{*}$-closed but not conversely. B. Chevreau and J. Esterle [6] proved the following interesting result.

Lemma 2.1. Let $\mathcal{S}$ be a weak*-closed subspace of $\mathcal{B}(\mathcal{H})$ with property $\left(\mathbf{A}_{1 / n}\right)$ for some $n \in \mathbf{N}$. Then $\mathcal{S}$ is WOT-closed.

The following elementary Lemma comes from [4, Lemma 9.15].

Lemma 2.2. Suppose that $n \in \mathbf{N}, T \in \mathcal{B}(\mathcal{H})$ and $\mathcal{S}$ is a linear subspace of $\mathcal{B}(\mathcal{H})$. Then the $n$ fold direct sum $T^{(n)}$ belongs to $\operatorname{Ref}\left(\mathcal{S}^{(n)}\right)$ if and only if whenever $\left\{x_{1} \ldots x_{n}\right\}$ and $\left\{y_{1} \ldots y_{n}\right\}$ are sequences from $\mathcal{H}$ such that $\sum_{j=1}^{n}\left[x_{j} \otimes y_{j}\right]=0$, we have $\sum_{j=1}^{n}\left\langle T x_{j}, y_{j}\right\rangle=0$. Moreover, $T$ belongs to the WOT-closure of $\mathcal{S}$ if and only if the $n$-fold direct sum $T^{(n)}$ belongs to $\operatorname{Ref}\left(\mathcal{S}^{(n)}\right)$, for every positive integer $n$. 
THEOREM 2.3. Let $\mathcal{S}$ be a weak*-closed subspace of $\mathcal{B}(\mathcal{H})$ with property $\left(\mathbf{A}_{1 / n}\right)$. Then $\mathcal{S}$ is $(2 n+1)$-reflexive.

Proof. By Lemma 2.1, $\mathcal{S}$ is WOT-closed. Much of the proof is based on ideas of [4]. Suppose the $(2 n+1)$-fold direct sum $T^{(2 n+1)}$ belongs to $\operatorname{Ref}\left(\mathcal{S}^{(2 n+1)}\right)$ for some $T$ in $\mathcal{B}(\mathcal{H})$. We have to show that $T$ belongs to $\mathcal{S}$. By Lemma 2.2, it suffices to show that the implication

$$
\sum_{j=1}^{k}\left[x_{j} \otimes y_{j}\right]=0 \Rightarrow \sum_{j=1}^{k}\left\langle T x_{j}, y_{j}\right\rangle=0
$$

holds for all integers $k$. We proceed by induction on $p$. By the hypothesis, we know that (5) is satisfied for $k \leq(2 n+1)$. Assume that (5) has been proven for all $2 n+1 \leq k<p$ and let $x_{1}, x_{2}, \ldots, x_{p}, y_{1}, y_{2}, \ldots, y_{p}$ in $\mathcal{H}$ satisfy the relation

$$
\sum_{j=1}^{p}\left[x_{j} \otimes y_{j}\right]=0
$$

Since $\mathcal{S}$ has property $\left(\mathbf{A}_{1 / n}\right)$, there exist sequences of vectors $\left\{u_{1}, \ldots, u_{n}\right\}$ and $\left\{v_{1}, \ldots, v_{n}\right\}$ in $\mathcal{H}$ such that

$$
\sum_{j=1}^{n}\left[u_{j} \otimes v_{j}\right]=\sum_{j=n+2}^{p}\left[x_{j} \otimes y_{j}\right]
$$

Since the equality $\sum_{j=1}^{n}\left[-u_{j} \otimes v_{j}\right]+\sum_{j=n+2}^{P}\left[x_{j} \otimes y_{j}\right]=0$ has $(p-1)$ terms, we have $\sum_{j=1}^{n}\left\langle-T u_{j}, v_{j}\right\rangle+\sum_{j=n+2}^{p}\left\langle T x_{j}, y_{j}\right\rangle=0$, or, equivalently,

$$
\sum_{j=1}^{n}\left\langle T u_{j}, v_{j}\right\rangle=\sum_{j=n+2}^{p}\left\langle T x_{j}, y_{j}\right\rangle .
$$

Furthermore, from (6) and (7) we have $\sum_{j=1}^{n+1}\left[x_{j} \otimes y_{j}\right]+\sum_{j=1}^{n}\left[u_{j} \otimes v_{j}\right]=0$. Thus it follows from the induction hypothesis that

$$
\sum_{j=1}^{n+1}\left\langle T x_{j}, y_{j}\right\rangle+\sum_{j=1}^{n}\left\langle T u_{j}, v_{j}\right\rangle=0
$$

since $k$ has $(2 n+1)$ terms. Consequently, by Lemma $2.2, T \in \mathcal{S}$. Hence the proof is complete.

We recover a result in [4] as a special case.

COROLlary 2.4. Suppose $\mathcal{S}$ is a weak*-closed subspace of $\mathcal{B}(\mathcal{H})$ with property $\left(\mathbf{A}_{1}\right)$. Then $\mathcal{S}$ is 3-reflexive.

The following proposition improves a result of A. Loginov and V. Shulman.

Proposition 2.5. Suppose $\mathcal{S}$ is a weak ${ }^{*}$-closed subspace of $\mathcal{B}(\mathcal{H})$ and has property $\left(\mathbf{A}_{1 / n}\right)$ and suppose $\mathcal{S}$ is $n$-reflexive. Then every weakly-closed subspace of $\mathcal{S}$ is $n$-reflexive, where $n$ is $a$ positive integer, (i.e., $\mathcal{S}$ is hereditarily n-reflexive). 
Proof. Suppose $\mathcal{M}$ be a weakly closed subspace of $\mathcal{S}$ and let the $n$-fold direct sum $T^{(n)}$ belong to $\operatorname{Ref}\left(\mathcal{M}^{(n)}\right)$. We have to show $T$ belongs to $\mathcal{M}$. It suffices to show the impliction $T \in \operatorname{Ref}_{E_{n}}(\mathcal{M}) \Rightarrow T \in \mathcal{M}$, where $E_{n}=E+E+\ldots+E$ ( $n$ summands). Assume that $T \in \operatorname{Ref}_{E_{n}}(\mathcal{M})$ and $T \notin \mathcal{M}$. Then $T \notin \mathcal{M}=\left(\mathcal{M}^{\perp}\right)_{\perp}$. Thus there exists $\varphi$ in $\mathcal{M}^{\perp}$ such that $\varphi(T) \neq 0$. Since $\mathcal{S}$ has property $\left(\mathbf{A}_{1 / n}\right)$, there exist sequences of vectors $\left\{x_{1}, \ldots, x_{n}\right\}$, $\left\{y_{1}, \ldots y_{n}\right\}$ in $\mathcal{H}$ such that

$$
\varphi-\sum_{i=1}^{n}\left[x_{i} \otimes y_{i}\right] \in \mathcal{S}^{\perp}
$$

Thus $\varphi \in \mathcal{M}^{\perp} \cap E_{n}$ and $\sum_{i=1}^{n}\left\langle T x_{i}, y_{i}\right\rangle \neq 0$. Thus we have $T \notin\left(\mathcal{M}^{\perp} \cap E_{n}\right)_{\perp}=\operatorname{Ref}_{E_{n}}(\mathcal{M})$. Consequently, $T \in \mathcal{M}$. Hence the proof is complete.

3. The Class $\mathcal{C}_{0}$. Recall that a completely nonunitary contraction $T \in \mathcal{B}(\mathcal{H})$ (on a separable Hilbert space $\mathcal{H}$ ) is an operator of class $\mathcal{C}_{0}$ if $u(T)=0$ for some $u \in \mathbf{H}^{\infty}, u \neq 0$. The simplest operators of class $\mathcal{C}_{0}$ are the Jordan Blocks $S(\Theta)$, with $\Theta \in \mathbf{H}^{\infty}$ an inner function, defined by

$$
\mathrm{S}(\Theta)=\left(\mathrm{S}^{*} \mid\left(\mathbf{H}^{2} \ominus \Theta \mathbf{H}^{2}\right)\right)^{*}
$$

where $S$ is the unilateral shift. It is known [3] that every operator $T$ of class $\mathcal{C}_{0}$ is quasi-similar to a Jordan operator $\mathrm{S}=\oplus_{i} \mathrm{~S}\left(\Theta_{i}\right)$, where the values of $i$ are ordinal numbers and the inner function are subject to the conditions $\Theta_{i}=1$ for some $i \geq 0, \Theta_{i}$ divides $\Theta_{j}$ whenever $i \geq j$ and $\Theta_{i}=\Theta_{j}$ whenever $\operatorname{card}(i)=\operatorname{card}(j)$. We start this section with the following Lemma from [7, Proposition 6].

Lemma 3.1. For an inner function $\Theta$, the weak*-closed algebra $\mathcal{A}_{\mathcal{S}(\Theta)}$ generated by 1 and $\mathcal{S}(\Theta)$ has property $\left(\mathbf{A}_{1,2}\right)(1)$.

THEOREM 3.2. Every operator of class $\mathcal{C}_{0}$ is 2-reflexive.

Proof. Suppose $T \in \mathcal{C}_{0}$. Then $T$ is quasi-similar to $\mathrm{S}=\oplus_{i} \mathrm{~S}\left(\Theta_{i}\right)$. Since $\mathcal{A}_{\mathrm{S}\left(\Theta_{i}\right)}$ has property $\left(\mathrm{A}_{1,2}\right)(1), \mathcal{A}_{\mathrm{S}\left(\Theta_{1}\right)}$ is weakly closed and 2-reflexive [4, Proposition 9.17], for $i=1,2, \ldots$ This implies that $\mathcal{A}_{\mathrm{S}\left(\Theta_{i}\right)} \oplus \mathcal{A}_{\mathrm{S}\left(\Theta_{2}\right)} \oplus \ldots$ is 2-reflexive. Since $\mathcal{A}_{\mathrm{S}}=\mathcal{A}_{\mathrm{S}\left(\Theta_{1}\right) \oplus \mathrm{S}\left(\Theta_{2}\right) \oplus \ldots}$ is contained in $\mathcal{A}_{\mathrm{S}\left(\Theta_{1}\right)} \oplus \mathcal{A}_{\mathrm{S}\left(\Theta_{2}\right)} \oplus \ldots, \mathcal{A}_{\mathrm{S}}$ is a weakly closed and has property $\left(\mathbf{A}_{1,2}\right)(1), \mathcal{A}_{\mathrm{S}}$ is 2-reflexive [Propostion 2.5]. Thus $\mathrm{S}=\oplus_{i} \mathrm{~S}\left(\Theta_{i}\right)$ is 2-reflexive. Since $T$ and $\mathrm{S}$ are quasi-similar operators of class $\mathcal{C}_{0}, T$ is 2-reflexive [3, Collary 3.6]. Hence the proof is complete.

An operator $T \in \mathcal{B}(\mathcal{H})$ is algebraic if $p(T)=0$ for some polynomial $p$.

COROllaRY 3.3. Every algebraic operator $T \in \mathcal{B}(\mathcal{H})$ with $\|T\| \leq 1$ is 2-hyperreflexive. Moreover, every algebraic operator with property $\left(\mathbf{A}_{2}\right)$ is hyperreflexive.

Proof. It is known that every algebraic operator with property $\left(\mathbf{A}_{2}\right)$ is reflexive [5, Corollary 6]. By [11, Theorem 3.14], every reflexive algebraic operator is hyperreflexive. Hence we have the corollary. 
4. Strictly $n$-separating vectors. In this section we study the relationship between $n$ hyperreflexity and strictly $n$-separating vectors. The following lemma comes from [11, Theorem 4.10].

LEMMA 4.1. Let $\mathcal{S}$ be a norm closed linear subspace of $\mathcal{B}(\mathcal{H})$. Suppose $\mathcal{S}$ has a strictly separating vector $e$ and a strictly separating closed subspace $\mathcal{M}$ such that $\overline{(\mathcal{S M})}=$ $\overline{s p}\{S x: S \in \mathcal{S}, x \in \mathcal{M}\}$ satisfies $\overline{(\mathcal{S M})} \cap \mathcal{S} e=\{0\}$ and $\overline{(\mathcal{S M})}+\mathcal{S e}$ is norm closed. Then $\mathcal{S}$ is hyperreflexive.

THEOREM 4.2. Let $\mathcal{S}$ be a norm closed linear subspace of $\mathcal{B}(\mathcal{H})$. Suppose $\mathcal{S}$ has a strictly $n$ separating vector. Then $\mathcal{S}$ is $(n+1)$-hyperreflexive for some $n \in \mathbf{N}$.

Proof. Suppose $\mathcal{S}^{(n)}$ has a strictly separating vector $e=\left(e_{1}, e_{2}, \ldots, e_{n}\right)$. We show that $\mathcal{S}$ has the $n$-dimensional strictly separating subspace $\mathcal{M}$. Suppose $=\overline{\operatorname{span}}\left\{e_{1}, e_{2}, \ldots, e_{n}\right\}$. We must show that $\mathcal{S} \rightarrow \mathcal{S} \mid \mathcal{M}$ is injective and $\mathcal{S} \mid \mathcal{M}$ is norm closed. Since $\mathcal{S}^{(n)}$ has a strictly separating vector $e=\left(e_{1}, e_{2}, \ldots, e_{n}\right)$.

$$
\left\|S^{(n)} e\right\| \geq \delta\left\|S^{(n)}\right\| \text { where } \delta>0 .
$$

Thus

$$
\begin{aligned}
\|S\|=\left\|S^{(n)}\right\| & \leq \frac{1}{\delta}\left\|S^{(n)} e\right\| \\
& =\frac{1}{\delta}\left\|S^{(n)}\left(e_{1}, e_{2}, \ldots, e_{n}\right)\right\| \\
& =\frac{1}{\delta}\left\|\left(S e_{1}, S e_{2}, \ldots, S e_{n}\right)\right\| \\
& =\frac{1}{\delta}\left(\sum_{i=1}^{n}\left\|S e_{i}\right\|^{2}\right)^{\frac{1}{2}} \\
& \leq \frac{\sqrt{n}}{\delta} \operatorname{Max}_{1 \leq i \leq n}\left\|S e_{i}\right\| .
\end{aligned}
$$

Then we have

$$
\|S \mid \mathcal{M}\| \geq \operatorname{Max}_{1 \leq i \leq n}\left\|S e_{i}\right\| \geq \frac{\delta}{\sqrt{n}}\|S\| .
$$

We define $\pi: \mathcal{S} \rightarrow \mathcal{S} \mid \mathcal{M}$ by $\pi(S)=S \mid \mathcal{M}$. Then we have

$$
\|\pi(S)\| \geq \frac{\delta}{\sqrt{n}}\|S\|=\beta\|S\|, \text { where } \beta=\frac{\delta}{\sqrt{n}}>0 .
$$

Thus $\pi$ is injective and ran $\pi$ is norm closed. Let $f=e \oplus 0 \in \mathcal{H}^{(n+1)}$. Then $f$ is a strictly separating vector for $\mathcal{S}^{(n+1)}$. Set

$$
\mathcal{G}=\{\underbrace{0 \oplus \ldots \oplus 0}_{(n)} \oplus m \mid m \in \mathcal{M}\} \subset \mathcal{H}^{(n+1)} .
$$


Then $\mathcal{G}$ is a strictly separating subspace for $\mathcal{S}^{(n+1)}$. We also note that $\mathcal{S}^{(n+1)} f \cap\left(\mathcal{S}^{(n+1)} \mathcal{G}\right)=\{0\}$ and $\mathcal{S}^{(n+1)} f+\left(\mathcal{S}^{(n+1)} \mathcal{G}\right)$ is norm closed. By Lemma $4.1, \mathcal{S}^{(n+1)}$ is hyperreflexive. Hence $\mathcal{S}$ is $(n+1)$-hyperreflexive.

Corollary 4.3. Suppose $\mathcal{S}$ is a norm closed linear subspace of $\mathcal{B}(\mathcal{H})$ and $\mathcal{S}$ has a finite dimensional strictly separating subspace $\mathcal{M}$. Then there is a positive integer $n$ such that $\mathcal{S}$ is $n$ hyperreflexive.

Proof. We can choose from unit sphere of $\mathcal{M}$ a finite collection of vectors $v_{1}, \ldots, v_{k}$ so that $\left(v_{1}, \ldots, v_{k}\right)$ is a strictly $k$-separating vector for $\mathcal{S}$. It follows from the above theorem that there is a positive integer $n(>k)$ such that $\mathcal{S}$ is $n$-hyperreflexive. Hence the proof is complete.

A subalgebra $\mathcal{A} \subset \mathcal{B}(\mathcal{H})$ is said to be strictly cyclic if $\mathcal{A} x=\mathcal{H}$, for some vector $x$ in $\mathcal{H}$.

COROLlaRY 4.4. Suppose $\mathcal{A}^{(n)}$ is a strictly cyclic abelian algebra. Then $\mathcal{A}$ is $(n+1)$ hyperreflexive, for some $n \in \mathbf{N}$.

Proposition 4.5. A weak*-closed subspace $\mathcal{S}$ has a strictly n-separating vector. Such as $\mathcal{S}$ has property $\left(\mathbf{A}_{1 / n}\right)(n r)$, for some $r>1$ and $n \in \mathbf{N}$.

Proof. Suppose $\mathcal{S}$ has a strictly $n$-separating vector. Then $\mathcal{S}^{(n)}$ has a strictly separating vector. Thus $\mathcal{S}^{(n)}$ has property $\left(\mathbf{A}_{1}\right)(r)$. By Proposition $7.3(1)$ of [1], $\mathcal{S}$ has a property $\left(\mathbf{A}_{1 / n}\right)(n r)$. The proof is complete.

In the following example, $\mathcal{S}^{(2)}=\{S \oplus S \mid S \in \mathcal{S}\}$ has a strictly separating subspace $\mathcal{M}$ but $\mathcal{S}^{(2)} \mathcal{M}$ is not norm closed.

Example 4.6. Suppose $\mathcal{S}$ is a norm closed linear subspace of $\mathcal{B}(\mathcal{H})$ and $\mathcal{S}$ has a strictly separating vector e. Let $f \in \mathcal{H}$ and $\mathcal{M}=s p\{e \oplus 0,0 \oplus f\}$. Then we have

$$
\begin{gathered}
\left\|S^{(2)} \mid \mathcal{M}\right\| \geq\left\|S^{(2)}(e \oplus 0)\right\|=\|S e\| \\
\geq \varepsilon\|S\|=\varepsilon\left\|S^{(2)}\right\| .
\end{gathered}
$$

Thus $\mathcal{M}$ is a strictly separating subspace for $\mathcal{S}^{(2)}$. But since

$$
\mathcal{S}^{(2)} \mathcal{M}=\left\{S_{1} e \oplus S_{2} f \mid S_{1}, S_{2} \in \mathcal{S}\right\}=\mathcal{S} e \oplus \mathcal{S} f
$$

$\mathcal{S}^{(2)} \mathcal{M}$ is not norm closed.

Acknowledgements The author would like to thank Professors Don Hadwin and Eric A. Nordgren for their valued suggestions and also wishes to express gratitude for their support to the KOSEF, 1997 and KOSEF 94-0701-02-01-3.

\section{REFERENCES}

1. E. A. Azoff, On finite rank operators and preannihilators, Mem. Amer. Math. Soc. 357 (1986).

2. $\mathrm{H}$. Bercovici, Operator theory and arithmetic in $H^{\infty}$, (Amer. Math. Soc., 1988).

3. H. Bercovici, C. Foias and B. Sz-Nagy, Reflexive and hyperreflexive operators of class $\mathrm{C}_{0}$, Acta Sci. Math. (Szeged) 43 (1981), 5-13. 
4. H. Bercovici, C. Foias and C. Pearcy, Dual algebra with applications to invariant subspaces and dilation theory, CBMS Conf. Ser. in Math. No. 56 (Amer. Math. Soc., 1985).

5. H. Bercovici, H. Kim and C. Pearcy, On reflexivity of operators, J. Math. Anal. Appl. 126 (1987), 316-323.

6. B. Chevreau and J. Esterle, Pettis'lemma and topological properties of dual algebras, Michigan Math. J. 34 (1987), 141-146.

7. K. Choi, Y. Jo and I. Jung, Dual operator algebras generated by a Jordan operator, Kyungpook Math. J. 34 (1994), 43-51.

8. K. Choi, I. Jung and B. Kim, On weak dilation of a representation, Houston J. Math. 22 (1996), 341-355.

9. L. Ding, On strictly separating vectors and reflexivity, Integral Equations Operator Theory 19 (1994), 373-380.

10. L. Ding, On a pattern of reflexivite operator spaces, Proc. Amer. Math. Soc., to appear.

11. D. Hadwin, Compression, graphs and hyperreflexivity, J. Funct. Anal., to appear.

12. D. Hadwin and E. Nordgren, Reflexivity and direct sums, Acta Sci. Math. (Szeged) 55 (1991), $181-197$

13. J. Kraus and D. Larson, Some applications of a technique for constructing reflexive operator algebras, J. Operator Theory 13 (1985), 227-236.

14. A. I. Loginov and V. S. Shulman, On hereditary and intermediate reflexivity of $w^{*}$-algebras, Izv. Akad. Nauk SSSR Ser. Math. 396 (1975), 1260-1273.

15. B.Sz-Nagy and C. Foias, Harmonic analysis of operators on Hilbert space (North-Holland, 1970).

16. K. Takahashi, On the reflexivity of contractions with isometric parts, Acta Sci. Math. (Szeged) 53 (1989), 147-152.

Department of Mathematics

College of National Sciences

KYUNGPOOK NATIONAL UNIVERSITY

TAGUE, 702-702, S. Korea
Present address

Department of Mathematics

College of Natural Sciences

Pohang University of Science and Technology

Pohang, 790-784, S. Korea 